\title{
Comprehensive analysis clinical effectiveness of Tirofiban for patients with non-ST-elevation acute coronary syndrome.
}

\author{
Jianfei Zheng ${ }^{1,2}$, Xudong Xiang1,2,*, Huixia Li $^{3}$, Bing Xiao', Ting Yuan ${ }^{1}$, Xun Gong ${ }^{1}$, Shuo Yao ${ }^{1}$ \\ ${ }^{1}$ Department of Emergency Medicine, Second Xiangya Hospital, Central South University, Changsha, Hunan, PR China \\ ${ }^{2}$ Emergency Medicine and Difficult Diseases Institute, Central South University, Changsha, Hunan, PR China \\ ${ }^{3}$ Department of Child Health Care, Hunan Provincial Maternal and Child Health Care Hospital, Changsha, Hunan, \\ PR China
}

\begin{abstract}
Background: To systematically assess the effectiveness and safety of tirofiban in patients with non-STelevation acute coronary syndromes (NSTE-ACS).

Methods: We search the online databases in both English and Chinese from inception to 2017 to collect randomized controlled trials about tirofiban for NSTE-ACS patients in early medicine conservative treatment. Two reviewers independently screened literature according to the inclusion and exclusion criteria, extracted data, and assessed methodological quality of included studies.

Results: A total of 10 randomized controlled trials were enrolled involving 6405 patients. The results of meta-analysis showed that: (1) The incidences of cardiovascular events of composite endpoints in the trial group were lower than that in the control group (during One week: relative risk $(R R)=0.55,95 \%$ confidence interval $(95 \% \mathrm{CI}$ : $0.40,0.74, \mathrm{P}<0.001)$ and the incidences of cardiovascular events of composite endpoints in the trial group were lower than that in the control group (during 30 days: $R R=0.45,95 \% C I 0.40,0.75, P<0.001)$. (2) The incidence of bleeding in the trial group was higher than that in the control group $(R R=1.31,95 \% C I$ : 1.00-1.72).

Conclusions: Compared with routine treatment alone, tirofiban has better therapeutic effects in early conservative treatment for patients with non-ST-elevation acute coronary syndromes, but the incidence of bleeding is relatively high.
\end{abstract}

Keywords: Tirofiban, Non-ST-elevation acute coronary syndrome, Randomized controlled trial, Meta-analysis Accepted on November 13, 2017

\section{Introduction}

Acute coronary syndrome (ACS) is a set of clinical syndromes whose pathological basis is coronary atherosclerotic plaque rupture or erosion, and then complete or incomplete occlusive thrombus form, including ST-segment elevation myocardial infarction (STEMI), non-ST-segment elevation myocardial infarction (NSTEMI) and unstable angina (UA) [1]. Even in the modern treatment conditions, the mortality, myocardial infarction rate and the re-admission rate of patients with acute coronary syndrome are still very high. ACS represents the performance of atherosclerosis of life-threatening. It is usually caused by the coronary atherosclerotic plaque rupture or erosion induced acute thrombosis. During the process of atherosclerotic plaque rupture, the release of inflammatory cytokines and inflammatory response occurs are critical pathologic physiology factors [2].

Percutaneous coronary intervention (PCI) is one of the most effective and common treatment of ACS. Especially for highrisk patients with ACS, clinical benefit of PCI is more pronounced $[3,4]$. The GP IIb/IIIa receptor antagonist approved listing used in ACS and PCI are in three categories: monoclonal antibody Fab fragment, synthetic peptides (such as eptifibatide), non-peptide biomimetic (such as tirofiban and lamifiban) [5]. In recent years, these drugs used in clinical practice, and have confirmed its efficacy in many clinical studies. However, the application of platelet glycoprotein IIb/ IIIa receptor antagonist in clinical practice still has some detail questions. In this study, we used Cochrane systematic review methods to make a Meta-analysis of randomized controlled trials of tirofiban for acute coronary syndrome patients undergoing percutaneous coronary intervention, in order to correctly assess the efficacy and safety.

\section{Methods}

\section{Literature search}

We search the databases in both English and Chinese, including The Cochrane Library (Issue 9, 2017), PubMed, 
EMbase, CNKI and WanFang Data from inception to 2017 to collect randomized controlled trials about tirofiban for NSTEACS patients in early medicine conservative treatment. We used the following medical subject heading terms and keywords to obtain potential studies: tirofiban, acute coronary syndrome, non-ST-elevation acute coronary syndrome, nonST-segment elevation myocardial infarction, unstable angina pectoris, and randomized controlled trial (RCT). The range of search was restricted in Chinese and English. We also reviewed the references lists of studies and relevant reviews.

\section{Criteria for inclusion}

Two reviewers independently screened literature according to the following inclusion and exclusion criteria: (1) study was conducted in patients with unstable angina pectoris/Non-STelevation acute myocardial infarction; (2) trial group received combined routine treatment (Clopidogrel, aspirin, low molecular heparin) and tirofiban intravenous infusion (load capacity: $4 \mu \mathrm{g} /(\mathrm{kg} . \mathrm{min})$; maintenance dose: $0.14 \mu \mathrm{g} /(\mathrm{kg} . \mathrm{min}))$, and control group received routine treatment only; (3) studies provided cardiovascular event rates. (4) Study should be RCT or cohort study; (5) the duplicates studies or with incomplete data or animal studies or ST elevated myocardial infarction were excluded; (6) the latest publication was included for the same study.

\section{Outcomes and data extraction}

The primary outcomes were incidences if cardiovascular events within 1 week and 30 days after treatment, including refractory myocardial ischemia, acute myocardial infarction, and sudden cardiac death. The second outcomes were incidences of bleeding events. JFZ and XDX extracted the following information: the first author, year of publication, age of trial and control group, male and female of both groups, sample size, prevalence of hypertension and diabetes.

\section{Assessment of quality}

We assessed the quality of included studies via the risk of bias tool recommended by Cochrane handbook [6]. This assessment tool consisted of the following seven items: random sequence generation; allocation concealment, blinding including study design and outcome assessment, selected reported data, incomplete data, and other potential bias. We identified each item as low risk, high risk or unclear risk according to reported results of each study when one item was judged as high risk.

\section{Statistical analysis}

We used relative risk to assess the effectiveness and safety of tirofiban in patients with NSTE-ACS. We firstly assessed the heterogeneity within studies through Chi-square test and I2 statistic [7]. Heterogeneity was confirmed when $\mathrm{P}<0.05$ or I $2>50 \%$. The random-effect model would be used to pool the results when there was heterogeneity, or else the fixed-effect model would be used. We conducted sensitivity analysis by excluding one studies each time. We used Begg's and Egger test to assess the publication [8,9]. All statistical analyses were completed on Stata 14.0 version software. $\mathrm{P}<0.05$ was treated as the significant level.

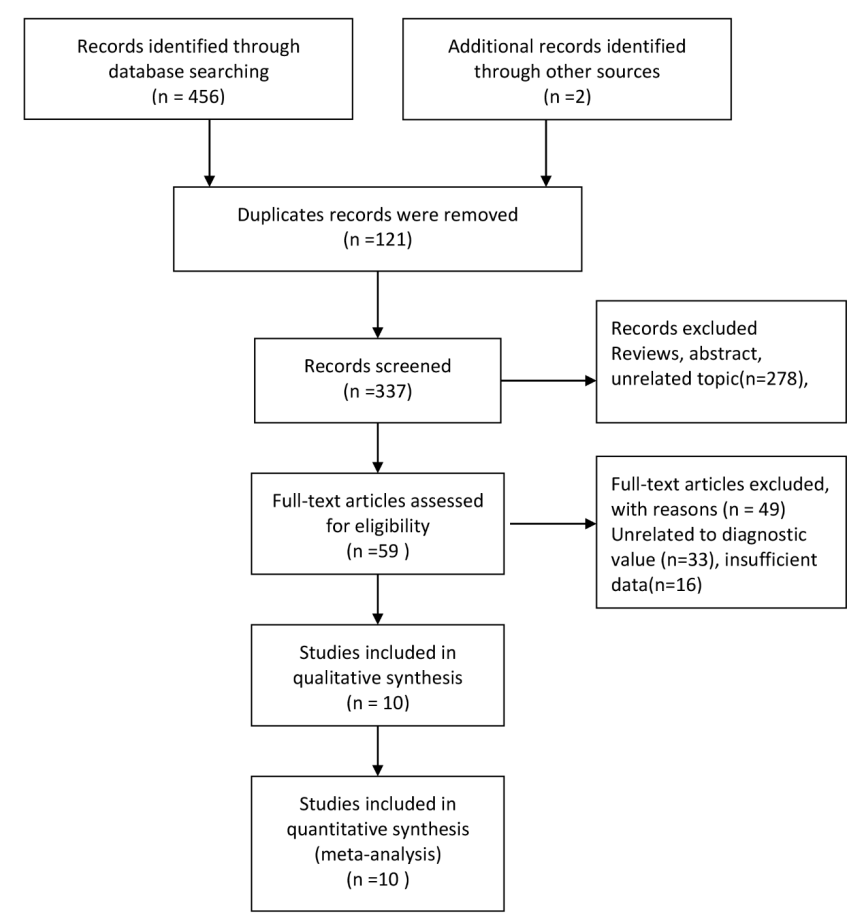

Figure 1. Flow chart of study selection.

\section{Results}

\section{Characteristics and quality of included study}

Our initial search returned 458 records, including 456 records through databases searching and 2 records identified through other sources. Finally, 10 studies were included in qualitative synthesis and quantitative synthesis [10-19]. The study selection was presented in Figure 1. The characteristics of included studies were presented in Table 1. Ten studies were published from 1998 to 2014. Six studies were from Asian population and the rest were from European population. The mean age was more than sixty-year old, and included 6405 study subjects. According to the scale, seven studies conducted study subjects through random number table methods, and three followed the order of registration. All studies used blinding methods. The quality of studies was relatively high.

\section{Pooled results}

Six studies reported the cardiovascular events incidences within one week. No significant heterogeneity was observed $(\mathrm{I} 2 \%=0.0, \mathrm{P}=0.466)$. The results suggested that the cardiovascular event incidence in trial group was lower than control group. There were obvious significant differences $(\mathrm{RR}=0.55,95 \% \mathrm{CI}: 0.40-0.74, \mathrm{P}<0.001)$. Nine studies reported the incidence of cardiovascular event for 30 days after treatment. The obvious heterogeneity was observed $(\mathrm{I} 2 \%=64.4 \%, \mathrm{P}=0.004)$. The results from random-effect model suggested that 30-day incidence of cardiovascular events in 
trial group was significantly lower than control group ( $R R=0.45$, 95\% $\mathrm{CI}$ : 0.40-0.75; Figure 2). Eight studies reported the incidence of bleeding, and no heterogeneity was found. We used the fixed-effect model to pool the data. The results found that the incidence of trial group was higher than control group $(\mathrm{RR}=1.31,95 \% \mathrm{CI}: 1.00-1.72)$.

Table 1. Characteristics of included study in the meta-analysis.

\begin{tabular}{|c|c|c|c|c|c|c|c|c|}
\hline Author & $\begin{array}{l}\text { Year } \\
\text { publication }\end{array}$ & of & $\begin{array}{l}\text { Age } \\
(\mathrm{T} / \mathrm{C})\end{array}$ & $\begin{array}{l}\text { Sex } \\
(M / F)\end{array}$ & & Sample size(T/C) & Hypertension & Diabetes \\
\hline Li et al. & 2014 & & $65.6 / 64.0$ & - & - & $114 / 112$ & $59 / 55$ & - \\
\hline Li et al. & 2004 & & $61.8 / 62.7$ & $26 / 13$ & $19 / 20$ & $39 / 39$ & $50 / 28$ & $21 / 57$ \\
\hline Li et al. & 2008 & & - & $22 / 21$ & $21 / 22$ & $43 / 43$ & $35 / 34$ & $27 / 31$ \\
\hline Gao et al. & 2009 & & $63.6 / 63.4$ & $20 / 20$ & $19 / 21$ & $40 / 40$ & $22 \% / 21 \%$ & $12 / 21$ \\
\hline PRISM & 1998 & & $62.5 / 62.4$ & $67.3 \% / 32.7 \%$ & $68.8 \% / 31.2 \%$ & $1616 / 1616$ & $54.2 \% / 54.6 \%$ & $20.4 \% / 22.2 \%$ \\
\hline PRISM-plus & 1998 & & $63.0 / 63.0$ & $67 \% / 33 \%$ & $68 \% / 32 \%$ & $1118 / 797$ & $52.5 \% / 56.0 \%$ & $23.5 \% / 24.0$ \\
\hline Kim & 2005 & & - & - & - & $59 / 61$ & - & - \\
\hline Bhattacharya & 2010 & & $62.6 / 62.7$ & $74 / 62$ & $89 / 76$ & $136 / 165$ & $113 / 145$ & $66 / 69$ \\
\hline Song et al. & 2007 & & - & - & - & $101 / 99$ & - & - \\
\hline Chen et al. & 2013 & & $64.5 / 62.8$ & $44 / 27$ & $42 / 29$ & $71 / 71$ & - & - \\
\hline
\end{tabular}

\begin{tabular}{llll} 
ID & RR $(95 \% \mathrm{Cl})$ & Weight \\
\hline Bhattacharya (2010) & $0.68(0.41,1.13)$ & 14.12 \\
Kim (2005) & $1.03(0.07,16.15)$ & 1.22 \\
PRISM (1998) & $0.82(0.63,1.06)$ & 19.67 \\
PRISMa (1998) & $0.86(0.66,1.11)$ & 19.82 \\
Song (2007) & $0.43(0.24,0.77)$ & 12.82 \\
Li (2014) & $0.48(0.33,0.71)$ & 16.85 \\
Li (2004) & $0.10(0.01,0.72)$ & 2.18 \\
Chen (2013) & $0.25(0.09,0.71)$ & 6.41 \\
Gao (2009) & $0.22(0.08,0.60)$ & 6.91 \\
Overall (I-squared = 64.4\%, $\mathrm{p}=0.004)$ & $0.55(0.40,0.75)$ & 100.00 \\
NOTE: Weights are from random effects analysis & & \\
\hline \multicolumn{1}{c|}{.0131} & 1 & 76.3 & \\
\hline
\end{tabular}

Figure 2. Comparison incidence of cardiovascular event risk for two groups within thirty days.

\section{Sensitivity analysis and publication bias}

The sensitivity analysis indicated that the results were stable (data not given). Egger and Beggar test indicated no obvious publication bias was observed $(\mathrm{Z}=1.360, \mathrm{P}=0.174 ; \mathrm{t}=2.290$, $\mathrm{P}=0.062$ ).

\section{Discussion}

The present study found that tirofiban has better therapeutic effects in early medicine conservative treatment for patients with non-ST-elevation acute coronary syndromes compared with routine drugs. However, patients who received combined tirofiban have higher risks of bleeding. Several ACS treatment guidelines recommend the early use of PCI treatment strategies [20,21]. However, during the pathogenesis of ACS and PCI intraoperative and postoperative have coronary endothelium damage, intimal exposed, platelet adhesion, activation and degranulation and the release of vasoactive substances such as ADP, 5-serotonin, etc., to induce further platelet aggregation activation, platelet glycoprotein lib/Ilia receptor conformational change, expose the fibrinogen binding sites of activated platelets, by the conformational change of the glycoprotein lib/Ilia receptor cross-linking with fibrinogen, and ultimately a large number of platelet aggregation, thrombosis, to cause ACS ischemic complications occurred, form stent thrombosis or mural thrombus, leading to the occurrence of adverse cardiac events [22]. From these pathogenesis of acute coronary syndrome ischemic complications, we can see that, platelet aggregation plays an important role. Platelet glycoprotein IIb/IIIa is one member of integrin adhesion receptors family, is the most abundant platelet membrane glycoprotein, its ligands are fibrinogen, von Wille brand factor, glass connected proteins, fibronectin protein. GP IIb/Ilia receptor antagonists by blocking the final common pathway of platelet aggregation, can effectively improve myocardial ischemia, reduce the incidence of mortality and myocardial infarction of patients [23]. With acute coronary syndrome. And the GP IIb/IlIa receptor is only found in the platelet and macrophage lineage, this feature enables specific treatment of its antagonists, without fearing of causing a wide range of side effects [24]. The appearance of platelet GPIIb/IIIa receptor antagonists is the milestone event in the history of ainti-platelet drug development [25]. Compared with the monoclonal antibody Fab fragment abciximab, small molecule GPI GP IIb/ IIIa receptor antagonist have a higher binding specificity, stronger role, and reversible combination, so, adverse reactions can be discontinued soon by immediately discontinued the drag, and as a synthetic peptide, themselves almost without antigen, do not cause allergic reactions, can be a long time or repeated treatment. 
In 2013, expert consensus recommended tirofiban for STEMI patients when patients did not receive enough double resist treatment [17]. Patients with high risk STEMI can be given to tirofiban before they are transferred to the emergency PCI. The GPI is not recommended for patients who have received both treatment and anticoagulant therapy.

This is consistent with the application of GPI drugs in acute ST-segment elevation myocardial infarction and treatment guidelines in 2015 [26]. For UA/NSTEMI patients, early invasive treatment was advised, if only use of aspirin and anticoagulation, tirofiban a combination therapy was recommended. If patients have double resistance treatment, but there are some risk factors such as diabetes, a positive troponin, and ST segment moving, and bleeding risk is low, it still can be used. For patients who are at high risk of bleeding and thrombosis, if they have been treated with double resistance, they are not recommended for early use of tirofiban.

Several limitations should be addressed. Firstly, we only search the published studies, some unpublished data may be ignored. Second, two studies were conducted in 1998, Clopidogrel was not widely used in clinical practice. Our results also found that heterogeneity existed within studies. The reason could be different intervention. Third, these results should be cautious because of different population setting. Further study is required.

In conclusion, early application of tirofiban was recommended for patients with non-ST-elevation acute coronary syndromes. However, the bleeding should be concerned.

\section{References}

1. Alonzo AA. Studying Acute Coronary Syndrome through the World Wide Web: Experiences and Lessons. JMIR Res Protoc 2017; 6: e182.

2. Katus H, Ziegler A, Ekinci O, Giannitsis E, Stough WG, Achenbach S, Blankenberg S, Brueckmann M, Collinson P, Comaniciu D, Crea F, Dinh W, Ducrocq G, Flachskampf FA, Fox K, Friedrich MG, Hebert KA, Himmelmann A, Hlatky M, Lautsch D, Lindahl B, Lindholm D, Mills NL, Minotti G, Mockel M, Omland T, Semjonow V. Early diagnosis of acute coronary syndrome. Eur Heart J 2017; 38: 3049-3055.

3. Ito S, Saio M, Suzuki T. Advanced atherosclerotic plaque as a potential cause of no-reflow in elective percutaneous coronary intervention: intravascular ultrasound and histological findings. J Invasive Cardiol 2004; 16: 669-672.

4. Kanaparti PK, Brown DL. Relation between coronary atherosclerotic plaque burden and cardiac enzyme elevation following percutaneous coronary intervention. Am J Cardiol 2000; 86:619-622.

5. Dery JP, Campbell ME, Mathias J, Pieper KS, Harrington RA, Madan M, Gibson CM, Tolleson TR, O'Shea JC, Tcheng JE. Complementary effects of thienopyridine pretreatment and platelet glycoprotein IIb/IIIa integrin blockade with eptifibatide in coronary stent intervention; results from the ESPRIT trial. Catheter Cardiovasc Interv 2007; 70: 43-50.

6. Higgins JP, Altman DG, Gotzsche PC, Juni P, Moher D, Oxman AD, Savovic J, Schulz KF, Weeks L, Sterne JA. The Cochrane Collaboration's tool for assessing risk of bias in randomised trials. BMJ 2011; 343: d5928.

7. Higgins JP, Thompson SG, Deeks JJ, Altman DG. Measuring inconsistency in meta-analyses. BMJ 2003; 327: 557-560.

8. Begg CB, Mazumdar M. Operating characteristics of a rank correlation test for publication bias. Biometrics 1994; 50: 1088-1101.

9. Egger M, Davey SG, Schneider M, Minder C. Bias in metaanalysis detected by a simple, graphical test. BMJ 1997; 315: 629-634.

10. Li FT, Wang ZJ, Wang JR, Yu XW. Evaluation on the role of tirofiban in high-risk non-ST-segment elevation acute coronary syndromes. South Chin Cardio Dis 2014; 20: 421-424.

11. Li F, Wang L, Po J. Clinical Investigation of Tirofiban in Treatment of Acute Coronary Syndrome. J Crit Care Inter Med 2004; 10: 72-74.

12. Li HL. Clinical observation of tirofiban in patients with Non-ST-elevation acute myocardial infarction. Chin J Mod Drug Appl 2008; 2: 67-68.

13. Gao YX, Li L, Liu HL, Yuan YQ. Effects of tirofiban on non ST-segment elevationj acute myocardial infarction. J Zhenzhou Univ (Med) 2009; 44: 406-408.

14. PRISM. Inhibition of the platelet glycoprotein IIb/IIIa receptor with tirofiban in unstable angina and non-Q-wave MI. N Engl J Med 1998; 338: 1488-1497.

15. PRISM-PLUS. Inhibition of the platelet glycoprotein IIb/ IIIa receptor with tirofiban in unstable angina and non-Qwave MI. N Engl J Med 1998; 338: 1488-1497.

16. Sim DS, Jeong MH, Kim W, Rhew JY, Yum JH, Kim JH, Cho JG, Ahn YK, Park JC, Ahn BH, Kim SH, Kang JC. Long-term clinical benefits of a platelet glycoprotein IIb/ IIIa receptor blocker, abciximab (ReoPro), in high-risk diabetic patients undergoing percutaneous coronary intervention. Korean J Intern Med 2003; 18: 129-137.

17. Bhattacharya R, Pani A, Dutta D, Basak S, Gangopadhyay S, Das BS, Sarkar RN. Randomised controlled trial evaluating the role of tirofiban in high-risk non-ST elevation acute coronary syndromes: an East Indian perspective. Singapore Med J 2010; 51: 558-564.

18. Song Y. Evaluation on the safety and efficacy of tirofiban in the treatment of acute coronary syndrome. J Huazhong Univ Sci Technolog Med Sci 2007; 27: 142-144.

19. Chen JB. Effects and safety of tifrobin in patients with non ST-elevation elevation acute coronary syndromes. Chin Med Engineer 2013; 21: 174-175.

20. Graber MA, Dachs R, Darby-Stewart A. Is prasugrel more effective than clopidogrel in patients with acute coronary syndrome scheduled for PCI? Am Fam Physician 2008; 78: 1252-1253. 
21. Urasawa K, Tsutsui H. [PCI for the patients with acute coronary syndrome]. Nihon Rinsho 2006; 64: 709-714.

22. Puricel S, Adorjan P, Oberhansli M, Stauffer JC, Moschovitis A, Vogel R, Goy JJ, Muller O, Eeckhout E, Togni M, Wenaweser P, Meier B, Windecker S, Cook S. Clinical outcomes after PCI for acute coronary syndrome in unprotected left main coronary artery disease: insights from the Swiss Acute Left Main Coronary Vessel Percutaneous Management (SALVage) study. Euro Intervention 2011; 7: 697-704.

23. Bigalke B, Haap M, Stellos K, Geisler T, Seizer P, Kremmer E, Overkamp D, Gawaz M. Platelet glycoprotein VI (GPVI) for early identification of acute coronary syndrome in patients with chest pain. Thromb Res 2010; 125: e184-e189.

24. Bigalke B, Langer H, Geisler T, Lindemann S, Gawaz M. Platelet glycoprotein VI: a novel marker for acute coronary syndrome. Semin Thromb Hemost 2007; 33: 179-184.

25. De Luca G, Savonitto S, Van'T HA, Suryapranata H. Platelet GP IIb-IIIa Receptor Antagonists in Primary
Angioplasty: Back to the Future. Drugs 2015; 75: 1229-1253.

26. Kraft P, Schuhmann MK, Fluri F, Lorenz K, Zernecke A, Stoll G, Nieswandt B, Kleinschnitz C. Efficacy and Safety of Platelet Glycoprotein Receptor Blockade in Aged and Comorbid Mice With Acute Experimental Stroke. Stroke 2015; 46: 3502-3506.

\section{*Correspondence to}

Xudong Xiang

Department of Emergency Medicine

Second Xiangya Hospital

Central South University

PR China 